\title{
Uterine ultrasound and endometrial biopsy in tamoxifen users
}

\author{
P. Neven ${ }^{1} \cdot$ W. Froyman ${ }^{1} \cdot$ S. Timmerman ${ }^{1} \cdot$ D. Timmerman ${ }^{1}$
}

Received: 4 March 2020 / Accepted: 10 March 2020

(c) Springer Science+Business Media, LLC, part of Springer Nature 2020

Jeon et al. studied 821 tamoxifen users for factors associated with endometrial pathologies [1]. 70\% with an endometrial biopsy were asymptomatic, while all the other 246 women had abnormal vaginal bleeding. Of these 246, 53 had an endometrial polyp, while only 7 had "endometrial pathology" defined as atypical endometrial hyperplasia or cancer. The authors were surprised by the absence of an association between endometrial cancer and three factors at endometrial exploration: age, menopausal status, or duration of tamoxifen use. Tamoxifen induces an extra endometrial cancer in 1 per 1000 women per year of use [2]. The median time of tamoxifen use in this series was 800 days and probably too short to study such associations. Regarding endometrial polyps that indeed can develop much earlier during tamoxifen use [3], we have some concerns regarding findings and recommendations suggested in this paper.

First, despite endometrial polyps being equally distributed in the group with (30.6\%) and without vaginal bleeding (29.3\%), the presence of bleeding symptoms was one of the three factors independently associated with "endometrial pathology" which is strange, as in this group, $89 \%$ were benign endometrial polyps and only few with endometrial pathology as earlier defined. Also, which proportion of those 7 with an endometrial cancer was asymptomatic?

This comment refers to the article available online at https://doi. org/10.1007/s10549-019-05448-w.

P. Neven

patrick.neven@uzleuven.be

W. Froyman

wouter.froyman@uzleuven.be

S. Timmerman

stefan.timmerman@uzleuven.be

D. Timmerman

dirk.timmerman@uzleuven.be

1 Department of Obstetrics and Gynecology, Multidisciplinary Breast Centre and Leuven University Centre for Cancer Prevention (LUCK), University Hospitals Leuven, Louvain, Belgium
Next, and another concern is that most data were from endometrial sampling in 575 asymptomatic women. To better understand their conclusion, it might be helpful to know the reason for endometrial sampling with or without general anesthesia. Also, it would help the reader if the authors can confirm that most endometrial pathology results, also in those with a polyp removal, showed endometrial atrophy or did some have endometrial hyperplasia without atypia? Tamoxifen typically induces (sub)endometrial cysts at ultrasonography and cystically dilated endometrial glands at histology, often in the context of the so-called endometrial cystic atrophy or adenomyosis.

Finally, the authors propose to use a difference of $2.9 \mathrm{~mm}$ in endometrial thickness, another independent factor associated with "endometrial pathology" in this series, or an undefined "shape of the ultrasonic endometrial appearance" to trigger "in-depth endometrial assessment" on tamoxifen use. This might even imply that they here suggest a (regular) follow-up with uterine ultrasonography which for us is another concern. Knowing that tamoxifen-associated endometrial polyps are not precursors of malignancy [4], we previously stated that a large proportion of tamoxifen users without endometrial pathology at the start will develop such subclinical lesions and that (regular) screening asymptomatic tamoxifen users with ultrasonography for endometrial pathology will lead to unnecessary anxiety, overtreatment of asymptomatic endometrial lesions, and even compromise patients' compliance with a life saving drug as tamoxifen therapy [5]. First, do not harm and do not advise tamoxifen users neither regular ultrasonography for endometrial screening nor more gynecologic examinations like painful blind endometrial biopsies. Appropriate assessment of the uterine cavity is only required with abnormal vaginal bleeding on tamoxifen. 


\section{Compliance with ethical standards}

Conflict of interest The authors declare that they have no conflict of interest.

Ethical approval This article does not contain any studies with human participants performed by any of the authors.

\section{References}

1. Jeon J, Kim SE, Lee DY et al (2020) Factors associated with endometrial pathology during tamoxifen therapy in women with breast cancer: a retrospective analysis of 821 biopsies. Breast Cancer Res Treat 179:125-130

2. Fisher B, Costantino JP, Redmond CK et al (1994) Endometrial cancer in tamoxifen-treated breast cancer patients: findings from the National Surgical Adjuvant Breast and Bowel Project (NSABP) B-14. J Natl Cancer Inst 86:527-537

3. Neven P, De Muylder X, Van Belle Y et al (1989) Tamoxifen and the uterus and endometrium. Lancet 8634:375

4. Dal Cin P, Timmerman D, Van den Berghe I et al (1998) Genomic changes in endometrial polyps associated with tamoxifen show no evidence for its action as an external carcinogen. Cancer Res 58:2278-2281

5. Neven P, Vergote I (1988) Should tamoxifen users be screened for endometrial lesions? Lancet 351:155-157

Publisher's Note Springer Nature remains neutral with regard to jurisdictional claims in published maps and institutional affiliations. 